\title{
LA PLANIFICACIÓN TURÍSTICA: UN ASPECTO CLAVE PARA EL DESARROLLO SOSTENIBLE Y REGIONAL DE BOYACÁ
}

\author{
PLANNING ON TOURISM: AN IMPORTANT ISSUE FOR THE SUSTAINABLE AND \\ REGIONAL DEVELOPMENT OF BOYACÁ
}

Tannia Álvarez Meneses

\begin{abstract}
Resumen
El artículo presenta inicialmente una compilación de información pertinente a la política pública nacional y regional en torno al turismo, a raíz de la gran importancia que ha adquirido esta actividad en el desarrollo económico y social del país y del departamento de Boyacá. En esta búsqueda se evidencian las falencias en las que ha caído el desarrollo turístico, haciendo énfasis especial en lo relacionado con los débiles procesos de planificación y de gestión integrados, que realmente conduzcan a un desarrollo turístico sostenible y por lo cual se hace necesario ejecutar acciones concretas, a través de proyectos y programas claramente definidos que aporten al mejoramiento de la calidad de vida de la comunidad local. Por ello es necesario entrar a profundizar en lo que implican estos procesos de planificación en turismo, los cuales deben estar enmarcados por principios del desarrollo sostenible. Esta reflexión conduce a evidenciar como la Universidad Pedagógica y Tecnológica de Colombia, desde su misión educativa y formativa, aporta de manera significativa a la región y contribuye a la cualificación del talento humano regional y local, a partir de una propuesta académica pertinente como lo es la Especialización en Planificación del Turismo Sostenible.
\end{abstract}

Palabras Clave: Turismo, Planificación Turística, DesarrolloTurístico, Desarrollo Sostenible.

\begin{abstract}
This article refers to some relevant information concerning tourism politics at regional and national level, based on the great importance that this activity has acquired in the economic and social development of the state of Boyacá and through the country. In this research work we can observe some failures in which tourism development has fallen, doing some special emphasis on the weak processes of planning and integrated management that really lead to a tourist sustainable development and for this reason it becomes necessary to issue clear actions through projects and clearly define programs that help to improve the quality of life of the local community, being also necessary to deepen in what these tourism planning processes really involve and must be framed under laws of sustainable development. This previous reflection lead us to show how the Pedagogical and Technological University of Colombia supports in a significant way the region and contributes to the qualification of the regional and local human resources starting from the university vision and mission view and also from a relevant academic offer which is its Specialization in Planning of the Sustainable Tourism.
\end{abstract}

Key words: Tourism, tourism planning, tourism development, sustainable development. 


\section{Introducción}

El turismo, concebido inicialmente como una actividad netamente económica por el intercambio que este originaba entre la demanda -visitantes y la oferta turística -atractivos y establecimientos de servicios turísticos de un destino, medido solo a través de indicadores de crecimiento, como la llegada de turistas y los ingresos generados, ha puesto en tela de juicio los beneficios que realmente conlleva esta actividad.

Ante las tendencias del desarrollo sostenible y/o sustentable, entendido como un proceso evaluable mediante los criterios e indicadores de carácter ambiental, económico y social que tiende a mejorar la calidad de vida y la productividad de las personas, que se funda en las medidas apropiadas de preservación de equilibrio ecológico, protección del ambiente, y aprovechamiento de recursos naturales, de manera que no se comprometa la satisfacción de las necesidades de las generaciones futuras (HCU, 1988) citado por (Herrera et al., 2012), se ha obligado a los diferentes sectores económicos del mundo a tomar en cuenta sus principios de manera transversal, y a permear en su implementación tanto a los Estados, como a todas las empresas propias de cada sector económico. Es así como el turismo ha volcado su mirada ante este escenario y ha reorientado el planteamiento de sus actividades de desarrollo en el marco de la sostenibilidad.

El desarrollo sostenible empieza a tomar fuerza desde 1987, cuando ante la ONU se presenta el informe Brundtland "Nuestro Futuro Común", propuesto por Harlem Brundtland, primera ministra de Noruega, donde se considera por primera vez el término y se señala como "aquel que satisface las necesidades del presente sin comprometer las necesidades de las futuras generaciones" (Goodland, 1994), y se dimensiona el equilibrio entre lo económico, social y ecológico.
En 1992 se consolidan los tres principios fundantes del desarrollo sostenible, establecidos durante la Conferencia de las Naciones Unidas sobre el Medio Ambiente y el Desarrollo, la sostenibilidad ambiental, la sostenibilidad social y cultural y la sostenibilidad económica; desde ellos los países adquieren grandes compromisos, pero en la realidad muy pocos los asumen con responsabilidad.

Ante este escenario, el turismo hace lo suyo y guiados por estos principios en 1995 se realiza en Lanzarote, España la Conferencia Mundial del Turismo Sostenible, sobre la consideración de que el turismo "es una actividad ambivalente, dado que puede aportar grandes ventajas en el ámbito socioeconómico y cultural, mientras que al mismo tiempo contribuye a la degradación medioambiental y a la pérdida de la identidad local, por lo que debe ser abordado desde una perspectiva global". (Conferencia Mundial del Turismo Sostenible, 1995). El documento resultado de esa conferencia reúne 18 principios del turismo sostenible, realizando recomendaciones claras a los gobiernos estatales y regionales para que formulen acciones que los promuevan.

Estos principios evidencian una orientación a la preservación de los recursos naturales y culturales; pero ello implica un gran esfuerzo en crear $y$ desarrollar instrumentos de planificación y gestión integrados que den cuenta del desarrollo sostenible del turismo, de modo que sean apropiados por las regiones como herramienta para hacer frente a los riesgos que pueda traer el desarrollo turístico. Entre los riesgos se tienen: el crecimiento desmedido en unidades habitacionales, la contaminación de los escenarios naturales como playas, ríos, mares y nevados, el desperdicio y uso irracional de los recursos hídricos, la acumulación de basuras y desechos, así como el uso indiscriminado de recursos energéticos y la generación de trabajos poco dignos, que no llevan a un mejoramiento de la calidad de vida de la población local. 
Sin embargo, surge la inquietud en cómo aterrizar estos principios de sostenibilidad en el turismo; lo primero es la voluntad pública de generar políticas que los contemplen y sean las guías para el desarrollo turístico, pero a su vez definir modelos de gestión pública del turismo que conlleve a la formulación e implementación de acciones de planificación, donde intervengan comunidad local, empresarios y autoridades de la mano con la academia como aliado estratégico, quien debe contribuir al desarrollo de competencias en el talento humano en torno a la planificación y promover la consolidación de nuevas herramientas y modelos en el contexto regional, que sean factibles de implementar y que orienten el desarrollo del turismo sostenible.

Para alcanzar este gran objetivo, las instituciones educativas desde su misión asumen con responsabilidad el dar respuesta a las necesidades del entorno, motivando a la reflexión y a partir de estudios de investigación estructuran programas pertinentes a la región en busca de una alta cualificación del talento humano.

Asumiendo dicha responsabilidad, la Universidad Pedagógica y Tecnológica de Colombia, UPTC, ha propuesto un posgrado en Planificación del Turismo Sostenible, soportado por los principios del desarrollo sostenible, articulado con las políticas nacionales y regionales y con un enfoque teórico orientado hacia las tendencias investigativas del turismo. El propósito de éste posgrado es el de formar profesionales que promuevan procesos de planificación turística sostenibles, acordes con el desarrollo regional y local, a partir de la planificación y la investigación como fundamentos, para contribuir al mejoramiento de la calidad de vida de las comunidades.

\section{Marco de Referencia}

\subsection{Contexto del Turismo en Boyacá}

El departamento de Boyacá, situado en la Región
Andina, en el centro - oriente colombiano, cuenta con una superficie de $23.189 \mathrm{Km} 2$, región que reúne gran parte de la historia de la independencia de Colombia, considerándose como la cuna de la libertad nacional. Su población al año 2012 fue de 1.271.133 habitantes, según cifra arrojada por el Departamento Administrativo de Estadística, DANE, de cuya población el $55,28 \%$ es urbana y el 44,72\% rural (Gobernación de Boyacá, 2012). Este Departamento es reconocido, a nivel nacional e internacional, por su riqueza arquitectónica, histórica - cultural y natural, y se prevé como un destino turístico de clase mundial para el año 2015 (Gobernación de Boyacá 2012).

En su estructura política e institucional cuenta con la Secretaría de Cultura y Turismo la cual refuerza el trabajo realizado por la Administración Departamental, dada la importancia adquirida del sector turístico como eje transversal en el desarrollo económico, político, social y ambiental del Departamento y reconocido dentro de sus principales actividades económicas, al igual que la producción agrícola y ganadera, la explotación de minerales, la industria siderúrgica y el comercio.

El Departamento cuenta con 123 municipios y 13 provincias: Gutiérrez, Centro, Márquez, Neira, Occidente, Norte, Oriente, Ricaurte, Valderrama, Sugamuxi, Tundama, Lengupá y La Libertad que reúnen la potencialidad turística de la región bajo el reconocimiento del turismo como factor de desarrollo local, al ser identificados 69 municipios con vocación turística un poco más del $56 \%$ del total de los municipios; que han señalado el turismo como una línea estratégica al interior de sus planes de gobierno municipal, dato revelado por la Secretaría de Cultura y Turismo del Departamento en el año 2011.

La región boyacense en torno al turismo, concentra una gama de ventajas comparativas reveladas en su riqueza natural, biodiversidad cultural y sus antecedentes históricos, y patrimoniales, acompañadas del desarrollo de infraestructura y empresas turísticas que han creado una fuerte 
demanda hacia destinos como Tunja, Paipa, Villa de Leyva, Lago de Tota, Sierra Nevada del Cocuy, Chiquinquirá, Ráquira, Moniquirá y Tenza, entre otros. Se registra una marcada tendencia de crecimiento por el turismo receptivo de naturaleza, histórico cultural, de negocios, de salud y deportivo.

Ante este escenario tan prominente, vale la pena mencionar que el Departamento no solo está comprometido con el desarrollo de estrategias que incentiven el crecimiento de indicadores económicos, sino que resalta la importancia en fortalecer acciones encaminadas a la sostenibilidad, que vayan a la par con el desarrollo armónico de la región, al citar aspectos ambientales y socioculturales en los últimos planes de gobierno departamental. En este sentido, el gobierno departamental en alianza con la Fundación Centro para el Desarrollo Tecnológico de Comercio, Turismo y Servicios, FUNDECOMERCIO, elaboró y consolidó con la participación de la comunidad El Plan de Desarrollo Turístico Sostenible del departamento de Boyacá 2012-2022.

Éste plan refleja no solo el interés departamental por ser parte activa en procesos sostenibles $y$ sustentables, sino que considera en el turismo una fuente de desarrollo que incorpora la construcción de indicadores de desarrollo sostenible. Proceso que fue consolidado de la mano con la visión país 2032 y articulados con el Plan de Desarrollo Nacional 2010-2014 Prosperidad para Todos, en el cual se proyecta el desarrollo e innovación de algunos sectores emergentes, que aportarían a la competitividad nacional. Al ser el turismo uno de esos sectores, se verá fortalecido a partir del mejoramiento de sus procesos de logística, la inclusión de tecnologías de información y comunicación y la consolidación de nuevos productos turísticos. Estas metas trazadas teniendo en cuenta las necesidades del país cuyo fin es alcanzar altos niveles de desarrollo turístico nacional y ser reconocido en el ámbito internacional.
Adicionalmente, el gobierno nacional ha aportado al desarrollo turístico nacional por medio de una política pública: Plan Sectorial de Turismo 2011 2014, en la cual señala como objetivo central mejorar la competitividad de los servicios y destinos turísticos de Colombia, con el fin de hacer del turismo una estrategia de desarrollo sostenible para el país que contribuya a la generación de empleo, a la prosperidad de las regiones y a dinamizar el círculo virtuoso del ascenso social. Para ello se enfoca en el logro de objetivos específicos, como el de "Fortalecer la institucionalidad y la gestión pública del turismo a nivel nacional y regional", definiendo claramente la estrategia de promover en las regiones la planificación del desarrollo turístico (Ministerio de Comercio, Industria y Turismo, 2011).

La gestión pública deberá englobar sus pilares fundamentales relacionados con el desarrollo turístico en cuanto al nivel de capacitación de personas, actitud de la comunidad local, la calidad de sus atractivos y el fortalecimiento de la investigación en turismo. Con una política turística regional que promueva la inversión y el desarrollo empresarial, así como el fortalecimiento de su infraestructura turística y la generación de acciones de promoción que incremente la afluencia de visitantes. Todos estos aspectos apoyados por herramientas de planificación, que permitan orientar procesos de desarrollo sostenible. De manera que la sostenibilidad, como eje estratégico de La Política de Competitividad Turística Nacional, sea apropiada por las regiones, con el fin de enmarcar todos sus procesos de desarrollo en las dimensiones económica, ambiental y socio cultural, y que a su vez esté soportada por la posibilidad de ser aplicadas en todo destino y en las diversas tipologías del turismo, con la posibilidad de generar un impacto positivo en los niveles de satisfacción de los turistas y de la comunidad local. (Ministerio de Comercio, Industria yTurismo, 2009).

En Boyacá se identifica como potencialidad para su desarrollo el Agroturismo y Ecoturismo, Turismo cultural y de Aventura, y se crea su visión al año 2025 
como un departamento que: "estará dentro de los diez principales destinos ecoturísticos y culturales reconocidos a nivel latinoamericano", pero en la cual se reconoce una gran problemática regional, que indica una "Débil planificación turística en el Departamento", desde donde se desprende la estrategia "Adelantar proceso de planificación turística para el desarrollo sostenible, con metodologías del Vice ministerio de Turismo", que será posible mediante el desarrollo de acciones Como (Ministerio de Comercio, Industria y Turismo, 2009):

a. Revisión de información secundaria y levantamiento de información primaria sobre: atractivos turísticos, actualización de inventarios turísticos, proyecto para el diseño de perfil de mercado y/o investigación de mercado nacional/internacional, segmentación del mercado, información general del destino, infraestructura, superestructura, capacitación, uso de tecnología, planta turística.

\section{b. Formulación de objetivos y estrategias de planificación.}

c. Identificación y estructuración de dos proyectos básicos, priorizando: "Determinación de la capacidad de carga y planes de manejo de atractivos turísticos con metodologías pertinentes".

\section{d. Divulgación del plan.}

Es así como conducir al fortalecimiento de estos factores es aterrizar en procesos de planificación del turismo, que involucra la gestión de las organizaciones turísticas con sus indicadores financieros, de mercado, administrativos y de responsabilidad social empresarial con la gestión pública del turismo hacia el uso racional de los recursos sean estos naturales o culturales, acorde a las condiciones de las regiones donde se desarrolla y que soporta innegablemente procesos sostenibles.
Procesos de planificación, entendidos como el primer pasó hacia la competitividad del turismo, que se estiman como un diagnóstico donde se ve la necesidad de implementar estos procesos para poder establecer las metas, punto de partida de la medición. Anotando que "sin la existencia del convencimiento y comprensión del destino como empresa compleja, no es posible intentar procesos de medición de las diversas actividades y proyectos. Ello implica que el destino haya realizado procesos de planificación de la actividad turística que le permitan diagnosticar el estado del arte de las diversas variables que afectan la actividad." (Ministerio de Comercio, Industria y Turismo, 2008).

Boyacá ha procurado fortalecer el turismo, prueba de ello han sido sus planes de desarrollo departamental, ya sea el actual "Boyacá se Atreve 2012 - 2015", o el de sus antecesores que han mantenido viva esta actividad y han formulado acciones encaminadas a alcanzar un desarrollo local a partir del turismo como factor clave para el desarrollo económico y social.

En el gobierno actual se contemplan dos ejes estratégicos y claves: uno de ellos hace referencia a "Un Boyacá que se atreve a generar desarrollo económico sin atentar contra el medio ambiente", lo que evidencia el compromiso que el gobierno departamental adquiere con el trato al medio ambiente. Al interior de este eje se ven reflejados dos programas estratégicos que orientan su ejecución, estos son desarrollo sostenible y planificación territorial. Al respecto de este eje estratégico el gobierno dispondrá de herramientas que incentiven unas mejores prácticas del orden productivo, es decir sin discriminación de sector económico de la región. Adicionalmente, se cita la importancia del respeto por los recursos naturales esencia del turismo, los cuales se manejarán dentro de una sostenibilidad que permita un desarrollo económico, haciendo referencia a un aspecto más, que envuelve el desarrollo sostenible. Complementariamente, hace énfasis en la necesidad de proteger los ecosistemas e invita al 
sector productivo a asumir la responsabilidad social empresarial y la generación de empleo principalmente en donde se localizan los nodos productivos.

En el segundo eje:“Una economía que se atreve a generar valor agregado para potencializar su riqueza", está implícito el turismo en su programa estratégico "Turismo de Clase Mundial" con el lema "Boyacá y las siete maravillas un destino de clase mundial". Al respecto se hace evidente como el turismo se considera un factor determinante para el desarrollo económico y social del departamento y menciona la importancia de articular esta actividad con la política nacional de turismo y apunta a ser reconocido por su diversidad en atractivos, al reunirlos en el tema turismo naturaleza, cultural y religioso.

A partir de este programa estratégico se evidencia el planteamiento de acciones que apuntan al fortalecimiento de la actividad turística, sobre las tipologías del turismo naturaleza, cultural y religioso, en articulación con la política nacional de turismo. Estableciendo como metas su posicionamiento como el quinto destino turístico a nivel nacional a través de la consolidación de sus productos turísticos, sobre la base de las 7 maravillas de Boyacá (Lago de Tota, Sierra Nevada del Cocuy, occidente Pie de Monte Llanero, Paipa, Tunja y Villa de Leyva) en consonancia con la marca región y sus estrategias de promoción.

Se puede concluir que el "Plan de Desarrollo Boyacá se Atreve 2012 - 2015", centra sus acciones en el desarrollo sostenible y lo presenta como el pilar fundamental en el desarrollo de la economía, las necesidades de la población, sin comprometer el bienestar de las generaciones futuras, donde existe un equilibrio en el crecimiento económico, progreso social y sostenibilidad ambiental en el territorio (Gobernación de Boyacá, 2012). Se hace énfasis en el desarrollo endógeno, concebido desde una mirada de lo rural, buscando el desarrollo de la población campesina, lo mismo que propiciar las condiciones para garantizar la seguridad alimentaria de la población en el territorio, teniendo como elemento importante la sostenibilidad ambiental.

\subsection{La Planificación Turística}

El informe Brundtland presenta su relación entre crecimiento y desarrollo: "crecer es aumentar el tamaño como consecuencia de la asimilación o acumulación de los materiales, mientras que desarrollar significa expandir o realizar los potenciales o llevar a un estado mejor, mayor o más completo. Cuando algo crece, se agranda cuantitativamente, cuando algo se desarrolla, mejora cualitativamente". (Goodland, 1994, p. 17).

Si bien el Departamento de Boyacá ha hecho hincapié en el desarrollo del turismo, es necesario orientar adecuadamente este proceso con la implementación de acciones de planificación, que en primera instancia implican tener claridad en conceptos de crecimiento y desarrollo económico, de sostenibilidad y de comunidad.

Con lo anterior se refuerza la intención de promover un desarrollo sostenible a nivel regional, lo cual implica que los indicadores con los que se mida sean distintos a los tradicionales, como por ejemplo la ocupación hotelera y la densidad promedio (Herrera, Acle \& Paredes, 2012), por otros indicadores de desarrollo sostenible, cuyo objetivo primordial sea mejorar la calidad de vida de la población residente, a través de la actividad turística y así se favorezcan las condiciones sociales, ambientales y económicas de la región.

Pensar en formación en Planificación del Turismo Sostenible, hace referencia al compromiso con el desarrollo sostenible, que es un concepto esencial para alcanzar metas de desarrollo sin agotar los recursos naturales y culturales ni deteriorar el medio ambiente, como lo contempla la Ley 99 de 1993, Ley General del Ambiente en Colombia. "Si una comunidad cuenta con el tipo adecuado de recursos, quizá tenga la oportunidad de desarrollar una forma de turismo que reporte considerables 
beneficios a todos sus miembros. Para lograrlo el turismo comunitario debe planificarse y gestionarse de forma que mejore la calidad de vida de los residentes y proteja el medio ambiente local, tanto natural como cultural. La protección del medio ambiente y el éxito en el desarrollo turístico son inseparables"(Fernández, 1995, p.57).

No solo la empresa privada sino las Instituciones del Estado tienen la responsabilidad de estimular y apoyar la conservación de la naturaleza y de la cultura que son los recursos más importantes para el turismo. También es tarea del gobierno fijar las políticas, los planes y el marco jurídico necesarios para un control del turismo, de forma que este aporte beneficios reales sin crear problemas graves. A partir de las políticas gubernamentales se presenta la necesidad de estimular que los Planes de Ordenamiento Territorial lleven a su revisión por parte de las entidades municipales, con el fin de orientar en su estructura un conjunto de acciones político-administrativas y de planta física, que promuevan el desarrollo de su territorio, de acuerdo con las estrategias de desarrollo socioeconómico y en armonía con el medio ambiente y las tradiciones históricas y culturales; así se posibilita un desarrollo local de manera segura y sostenible propendiendo por el mejoramiento de la calidad de vida.

La tarea planificadora depende de la decisión y el compromiso de los actores regionales más representativos, incluidas las comunidades como principal célula para su funcionamiento. Se debe asumir con plena responsabilidad social. La acción planificadora tiene que estar "inmersa en el proceso de cambio profundo que sufre la estructura de un país poniendo el énfasis en la descentralización y en la participación de la comunidad organizada" (Fernández, 1995, p. 21) citado por (Álvarez, 2011). El turismo debe pugnar por un desarrollo sostenible de la actividad y no debe olvidarse que es un servicio inscrito en las actividades económicas y administrativas, pero que su base es lo social, lo antropológico, por lo que no se deben anteponer objetivos de crecimiento, sino también de desarrollo.

Es evidente que el turismo cuenta con el apoyo del gobierno departamental, al considerarlo un motor para el desarrollo económico y al contemplarlo en su plan estratégico con el objetivo claro de hacer del turismo una actividad que contribuya al desarrollo sostenible, bajo estándares de competitividad para posicionar a Boyacá como uno de los principales destinos turísticos sostenibles del país. Se resalta la importancia de la formación en turismo proponiendo los programas de fomento $y$ desarrollo para la investigación del sector turístico y el de formación y cualificación del talento humano para el turismo, teniendo como acciones específicas la formulación de la política pública departamental de turismo y el desarrollo de programas de formación en turismo y la creación de planes turísticos, respectivamente. (Gobernación de Boyacá, 2008).

El turismo es una actividad con potencial, pero hay que asumir esta tarea con responsabilidad, lo que implica incentivar la realización de procesos de planificación serios donde intervengan todos los actores del sistema turístico y no caer en el error de considerar solo el crecimiento, que lamentablemente permite el deterioro de los recursos naturales, pérdida de identidad cultural y vicios sociales entre otros. Los que tienen esa responsabilidad deben contar con los elementos para planificar el turismo, de forma sostenible.

\subsection{Especialización en Planificación del Turismo Sostenible}

Las instituciones educativas deben apoyar los procesos de planificación, ya sea por medio del fortalecimiento de la actividad a partir de la investigación formativa que pretende desarrollar la capacidad de indagar y comprender la problemática regional, así como generar un acercamiento a la investigación científica que aporta al sector objeto de estudio y promueve su desarrollo. 
En concordancia con los lineamientos estratégicos del Departamento y en pro de la articulación entre gobierno y academia, la UPTC como actor involucrado en el desarrollo del Departamento, considera en su Plan Maestro de Desarrollo Institucional 2007 - 2019 "que el destino de la región es una responsabilidad de carácter colectivo en donde la Universidad juega un papel fundamental para generar inteligencia social capaz de producir conocimiento apropiado al desarrollo y, además, propugnar por una activa socialización del concepto de calidad de vida y por una conciencia colectiva de pertenencia e identidad regionales", (Universidad Pedagógica y Tecnológica de Colombia, 2011).

De lo anterior se destaca el interés de la universidad en aportar significativamente a la satisfacción de las necesidades regionales, formulando estrategias encaminadas al fortalecimiento del Departamento, y de manera específica, en el programa "Cobertura con pertinencia", del Lineamiento Regionalización. Se plantea la necesidad de contribuir a la solución de problemas regionales e impulsar a sectores estratégicos de acuerdo con potencialidades particulares del área de influencia, promoviendo la creación y extensión de programas académicos y procesos investigativos al servicio de la región.

Es así como, de manera articulada con el Plan de Desarrollo Institucional, la UPTC ha contemplado la necesidad de formar planificadores turísticos como primer pasó para alcanzar la eficiencia en la gestión del turismo sostenible en el Departamento. Por ello ha planteado la Especialización en Planificación del Turismo Sostenible, la cual basa su enfoque teórico y conceptual en el desarrollo sostenible, el cual se consolida desde la mirada de tres principios fundantes, establecidos en La conferencia de las Naciones Unidas sobre el Medio Ambiente y el Desarrollo en 1992, a saber:

"La sostenibilidad ambiental: debe garantizar que el desarrollo sea compatible con el mantenimiento de los procesos ecológicos esenciales, de la diversidad biológica y los recursos naturales.

La sostenibilidad social y cultural: debe garantizar que el desarrollo sostenible aumente el control de los individuos y sus vidas, y que sea compatible con la cultura y los valores de las personas y refuerce la identidad de las comunidades.

La sostenibilidad económica: debe garantizar que el desarrollo sea económicamente eficiente, beneficie a todas las gentes de la región afectada y que los recursos sean gestionados de manera que se conserven para las generaciones futuras." (Espinoza, 2008, p.66).

La Especialización, siendo coherente con la fundamentación del turismo sostenible, contempla los ámbitos ambiental, sociocultural, y económico, desde la perspectiva de la planificación, así:

Desde lo ambiental: el turismo ha adoptado nuevas posibilidades de desarrollo generando nuevas tipologías, dejando de lado el turismo convencional y pasando a un turismo alternativo en el que la naturaleza y la biodiversidad son el centro de dicha actividad, que promueve la preservación de los recursos y del medio ambiente, compatibilizando la capacidad de carga y la sensibilidad de un entorno natural y cultural en su práctica, prevaleciendo políticas de sustentabilidad de destinos así como de responsabilidad social empresarial.

Desde lo social y cultural se evidencia la necesidad de fortalecer la identidad de los seres humanosy de su identidad cultural, a partir del reconocimiento de sus bienes culturales y ambientales contemplados en su patrimonio, que lo caracterizan y que los hacen únicos y desde donde se desarrollan los hechos turísticos, sea este tangible o intangible, presente o pasado. De ahí que el turismo propenda por la valoración, el respeto y la promoción de los valores culturales, sensibilizándonos en la existencia de la multiculturalidad presente en el mundo. El turismo juega un papel muy importante 
en la sociedad, pues mientras de una parte educa y sensibiliza para la valoración y el disfrute de los bienes culturales -costumbre, folclor, manifestaciones artísticas, formas de expresión-y goce de recursos naturales, de otro lado ofrece opciones para el disfrute del ocio, la recreación y el tiempolibre.

Desde lo económico el turismo da cuenta de un activo intercambio de bienes y servicios propios de esta actividad, generando la riqueza necesaria para motivar a los habitantes de una región a su desarrollo de manera tal que la adaptación de los "sistemas productivos (y también los servicios) sean respetuosos con la calidad del medio (Pastor, 2003).

Los anteriores elementos se integran de manera coherente al interior de una propuesta curricular que busca a través de sus graduados comprender la problemática de la actividad turística a nivel regional, local y global, con conocimientos en planificación y desarrollo sostenible y un sentido de responsabilidad social hacia la implementación de prácticas turísticas sostenibles como una estrategia del desarrollo humano en los territorios.

\section{Conclusiones}

La planificación turística es un proceso serio y responsable, el cual debe ser liderado por las autoridades locales, con la participación de todos los actores del turismo, incluyendo a la comunidad local; lo anterior con un aporte significativo de la academia en su compromiso con el desarrollo regional.

El turismo proporciona una mejora en la calidad de vida de la comunidad local cuando se desarrolla de forma sostenible, pues promueve la conservación de los recursos, el fortalecimiento de la identidad cultural, el desarrollo de prácticas empresariales enmarcadas con criterios de protección al medio ambiente para el uso racional, cuidadoso y respetuoso de los recursos naturales y culturales, tangibles e intangibles.

El desarrollo turístico debe ser concebido sobre los tres pilares fundamentales del desarrollo sostenible, lo ambiental, social-cultural y económico, y acompañado por procesos de planificación. En términos de planificación turística se hace necesario construir indicadores de desarrollo sostenible, que permitan conducir a un verdadero desarrollo turístico que impacte en mayores beneficios a la comunidad local.

La planificación turística puede ser implementada por las regiones a partir de diversos modelos, lo importante es que en su desarrollo conduzcan a la conservación y preservación de sus recursos sean estos naturales o culturales.

\section{Agradecimientos}

La autora agradece el apoyo de la Dirección de Investigaciones de la UPTC, brindado a través del proyecto SGI-991, el cual respondió al proyecto de investigación como docente de primer nombramiento. Igualmente se agradece al Grupo Muisuata, por su apoyo y experticia en la investigación en turismo.

\section{Referencias}

Álvarez, T. (2011). Planificación de Circuitos Turísticos. San Gil:Unisangil Editora.

Conferencia Mundial del Turismo Sostenible. (1995). Carta del Turismo Sostenible. Lanzarote, España.

Espinoza, L. (2008). Sostenibilidad reto principal en el desarrollo del sector turístico. Costa Rica: Editorial Tecnológica de Costa Rica.

Fernández, V. (1995). Planificación de Entidades Territoriales. Colombia: Universidad Externado de Colombia. 
Goodland, R. (1994). Desarrollo Económico Sostenible, avances sobre el informe Brundtland. Colombia:Uniandes.

Gobernación de Boyacá (2008). Plan de Desarrollo "Para Seguir Creciendo" 2008 - 2011. Tunja: Departamento de Planeación.

Gobernación de Boyacá (2012). Plan de Desarrollo "Boyacá se Atreve" 2012 - 2015. Tunja. Departamento de Planeación.

Herrera, B., Acle, R. \& Paredes, R. (2012). Evaluación de recursos para el aprovechamiento del turismo de naturaleza en el área natural protegida estatal "Sierra del Tentzo" Puebla, México. Revista de Investigación Desarrollo e Innovación, 3 (1), 8-17.

Ministerio de Comercio, Industria y Turismo, Viceministerio de Turismo. (2008). Sistema de Indicadores de Competitividad para el Turismo Colombiano. Bogotá.
Ministerio de Comercio, Industria y Turismo, Viceministerio de Turismo (2009). Convenio de Competitividad Turística Departamento de Boyacá Matriz de Compromisos 2009. Bogotá.

Ministerio de Comercio, Industria y Turismo, Viceministerio de Turismo. (2011). Plan Sectorial de Turismo 2011 - 2014. Bogotá: Departamento Nacional de Planeación.

Naciones Unidas. (1992). Declaración de Rio sobre Medio Ambientey Desarrollo. Río de Janeiro.

Pastor, A. (2003). Turismo, Cultura y Medio Ambiente. España:Universidad de Alicante.

Universidad Pedagógica y Tecnológica de Colombia (2011). Plan Maestro de Desarrollo Institucional 2007 -2019. Tunja. 